\title{
NOTAS SOBRE EL GÉNERO PHYTOLACCA (PHYTOLACCACEAE) EN MÉXICO*
}

\author{
JERZY RZEDOWSKI \\ Y \\ Graciela Calderón de Rzedowski \\ Instituto de Ecología, A.C. \\ Centro Regional del Bajío \\ Apartado postal 386 \\ 61600 Pátzcuaro, Michoacán
}

\section{RESUMEN}

El género Phytolacca es de los taxonómicamente difíciles y su arreglo clasificatorio está pendiente de una cuidadosa revaluación. El examen de más de 1100 números de colecta revela la existencia en nuestro país de cinco entidades más o menos razonablemente distintas: $P$. americana L., P. icosandra L., P. rivinoides Kunth \& Bouché, $P$. rugosa Braun \& Bouché y $P$. thyrsiflora Fenzl ex A. J. Schmidt.

Aunque varios autores dudaron acerca de la presencia de $P$. americana más al sur de la porción oriental de los Estados Unidos, el estudio de abundantes materiales obtenidos en las últimas décadas, señala que esta especie extiende su área de distribución a lo largo de los parajes más húmedos de la Sierra Madre Oriental, desde Nuevo León y Tamaulipas hasta el norte de Oaxaca. Sus poblaciones mexicanas muestran mayor variabilidad morfológica que la observada en el oriente estadounidense y en función de tal circunstancia se piensa que la especie pudo haberse originado en este país y colonizado con posterioridad territorios ecológicamente similares, ubicados más al norte.

$P$. heterotepala $\mathrm{H}$. Walt., diferenciada a base del perianto ligeramente zigomórfico, al parecer sólo representa casos de anomalía floral en individuos de $P$. icosandra y de $P$. rugosa.

$P$. icosandra y $P$. octandra $L$. se han estado distinguiendo entre sí en función del diferente número de estambres y del largo de la inflorescencia. En el centro y sur de México, sin embargo, existen muchas poblaciones que no pueden asignarse claramente a ninguna de estas dos especies $y$, en expectativa de un estudio detallado que defina esta situación, se considera apropiado reconocer un solo taxon.

Aunque $P$. rugosa diverge de los demás representantes regionales de Phytolacca en sus carpelos libres en el extremo apical, su ubicación y circunscripción taxonómica no es clara. En México se pueden separar dos conjuntos morfológica y ecológicamente algo diferentes, uno de ellos mucho más similar a $P$. icosandra que el otro y posiblemente afectado por una mayor influencia genética de esta última especie.

Entre los materiales revisados se encontraron varios ejemplares en los que se pone de manifiesto la tendencia hacia la ramificación de la inflorescencia en sus porciones inferiores. Tres de estos individuos no difieren en otros caracteres de $P$. americana, $P$. rivinoides y $P$. rugosa, respectivamente y se interpretan como variantes esporádicas de tales especies. Algunos posiblemente

* Trabajo realizado con apoyo económico del Instituto de Ecología, A.C. (cuenta 902-03), del Consejo Nacional de Ciencia y Tecnología, así como de la Comisión Nacional para el Conocimiento y Uso de la Biodiversidad. 
pertenecen a $P$. thyrsiflora Fenzl ex J. A. Schmidt, especie de distribución esencialmente sudamericana, pero cuya circunscripción está todavía por definirse.

A título de conclusiones, se propone que las futuras aproximaciones a la taxonomía de Phytolacca reduzcan su dependencia del cómputo del número de los estambres e incorporen con más énfasis el aspecto de las afinidades ecológicas de las plantas. Se recomienda asimismo prestar debida atención a la posibilidad de la incidencia de anomalías florales y, en función de la importancia de las aves como dispersores de sus semillas, se sugiere tomar en cuenta las posibles consecuencias genéticas de la propagación a mediana y larga distancia.

\section{ABSTRACT}

The genus Phytolacca is a taxonomically difficult one and its classificatory arrangement is pending a thorough reappraisal. A study of more than 1100 collection numbers shows the existence in Mexico of five more or less reasonably distinct entities: $P$. americana $L$., $P$. icosandra $L$., $P$. rivinoides Kunth \& Bouché, $P$. rugosa Braun \& Bouché and $P$. thyrsiflora Fenzl ex J. A. Schmidt.

Although several authors expressed their doubts concerning the presence of $P$. americana in Mexico, the study of abundant materials obtained in last decades reveals that this species extends its area along the moister places of the Sierra Madre Oriental, from Nuevo León and Tamaulipas to northern Oaxaca. Its Mexican populations are morphologically more variable than those of eastern United States and in such circumstances it is believed that the species may have originated in Mexico and subsequently colonized ecologically similar territories situated to the north.

$P$. heterotepala $\mathrm{H}$. Walt., distinguished on the basis of slightly zygomorphic perianths, only seems to comprehend cases of floral anomalies in individuals of $P$. icosandra and $P$. rugosa.

$P$. icosandra and $P$. octandra L. are usually differentiated from each other in the number of stamens and in the length of the inflorescence. However, in central and southern Mexico numerous populations exist, in which plants do not adjust to this separation. Thus, in the expectation of a detailed study of this situation, only one taxon is recognized.

Although $P$. rugosa differs from the remaining regional members of Phytolacca in its apically free carpels, its taxonomic situation and circumscription is not clear. In Mexico within this complex two groups of plants can be separated on the basis of their morphological and ecological affinities, one of them being much more similar to $P$. icosandra than the other, possibly under stronger genetic influence of the latter.

Among the examined material several specimens show the trend toward inflorescence branching in its lower part. Three of these specimens do not differ in other characters from $P$. americana, $P$. rivinoides and $P$. rugosa, respectively, and are interpreted as sporadical variants of these species. Several possibly belong to $P$. thyrsiflora Fenzl ex J. A. Schmidt, an essentially South American species, the cirumscription of which remains to be defined.

In terms of conclusions it can be proposed that future approximations to the taxonomy of Phytolacca reduce their dependence from the computation of stamen-numbers and incorporate more emphasis on the aspect of ecological affinities of the plants. More attention is also recommended to the possibility of incidence of floral anomalies and in view of the importance of birds as dispersers of Phytolacca seeds, the genetic consequences of long and medium-distance dispersal are to be considered.

En el transcurso de la elaboración del fascículo correspondiente a la familia Phytolaccaceae para la Flora del Bajío y de regiones adyacentes los autores encontraron varios problemas con respecto a la apropiada delimitación y nomenclatura de algunas especies de Phytolacca. En consecuencia hubo necesidad de revisar con detenimiento la 
literatura existente, así como los materiales de herbario disponibles en las colecciones mexicanas, además de realizar observaciones en poblaciones naturales de los representantes de este grupo de plantas en nuestro país.

Algunos de los resultados de tal ejercicio se presentan a continuación.

\section{Aspectos generales}

Como lo señalan varios autores, el género Phytolacca es sin duda de los taxonómicamente difíciles. Tal situación obedece en primera instancia al hecho de que los individuos de muchas de sus especies son notablemente similares entre sí en su aspecto general. Se diferencian de manera primordial con base en caracteres de la inflorescencia, de la flor y del fruto, en su mayoría de tipo cuantitativo, en los cuales a menudo presentan intensa variación, no pocas veces en forma paralela.

Para mayor abundamiento, no es rara la convivencia de dos o más taxa diferentes, por lo que la hibridación, así como la introgresión genética no parecen ser excepcionales. También es muy probable que la incidencia de estas últimas debe haber ido en incremento a raíz de las modificaciones ambientales propiciadas por las actividades del hombre (Fassett y Sauer, 1950). En su gran mayoría las especies de Phytolacca son plantas que prosperan en habitats inestables; en su condición original se comportan como elementos pioneros de la sucesión y su frecuente proliferación actual definitivamente obedece a la expansión de habitats perturbados y de la vegetación secundaria. Es común verlas en calidad de malezas ruderales.

A causa de todas estas circunstancias, se han multipicado, sobre todo en el siglo $\mathrm{XIX}$, las descripciones de una considerable cantidad de "especies nuevas" de este género, muchas de las cuales no representan sino poblaciones extremas de taxa cuyos intervalos reales de variación no se conocen bien.

En el primer intento de una revisión del conjunto, Walter (1909) reconoció 26 especies que en su totalidad muestran amplia distribución mundial, aunque se encuentran particularmente concentradas en la región neotropical. Más recientemente Nowicke (1969) redujo este número a 20 y anticipó la probable necesidad de una rebaja más drástica, basada en el examen crítico de varios casos complicados y dudosos. Para explicar la falta de nitidez en la definición de las especies de Phytolacca a menudo se ha invocado la existencia de flujos evolutivos, lo cual posiblemente es cierto en varios casos, pero por otro lado es evidente que todavía no se han logrado discernir bien las bases correctas del arreglo sistemático de los componentes de este género.

Sin duda alguna, el grupo requiere de una revaloración detallada a nivel global, fundamentada no solamente en criterios morfológicos y geográficos, sino también con ayuda de otras metodologías que puedan resultar apropiadas, como posiblemente podrían ser análisis citológicos, quimiotaxónomicos y de índole molecular.

Mientras tanto y en espera de tal enfoque monográfico, a los estudios florísticos regionales sólo les cabe extraer el mejor provecho posible del esquema clasificatorio actualmente vigente y apoyarse hasta donde resulte factible en el conocimiento de las poblaciones naturales para resolver las situaciones dudosas o conflictivas.

En el caso concreto del territorio de la República Mexicana, la revisión del amplio material de herbario (alrededor de 1800 especímenes, equivalentes a más de 1100 
números), que se ha ido acumulando en las últimas décadas, revela la existencia de cinco especies espontáneas básicas de Phytolacca, delineadas en forma más o menos clara. Estas entidades son esencialmente las mismas que distinguió Martínez-García (1984, 1986), aunque los que escriben discrepan en la identificación de una de ellas.

Los cinco taxa en cuestión son muy similares entre sí en las características vegetativas: todos corresponden a plantas herbáceas perennes robustas, a menudo manchadas de tonalidades moradas o purpúreas, glabras o casi glabras, con tallos gruesos, algo suculentos, así como hojas de tamaño y forma algo variables, pero que básicamente son elípticas, de margen entero y con frecuencia atenuadas en ambos extremos. Las diferencias fundamentales aparentemente se restringen a los rasgos de los órganos reproductores y se resumen en la siguiente clave, que puede ser útil para la identificación de la mayor parte de los especímenes.

1 Tépalos deciduos en la fructificación; gineceo con (10) 12 a 18 carpelos; inflorescencia hasta de 50 (70) $\mathrm{cm}$ de largo, glabra, pedicelos muy manifiestos, en fruto hasta de $15 \mathrm{~mm}$ de largo $P$. rivinoides

1 Tépalos persistentes en la fructificación; gineceo con (7) 8 a 10 (11) carpelos; inflorescencia hasta de $25(40) \mathrm{cm}$ de largo, frecuentemente papiloso-costrosa, pedicelos conspicuos a prácticamente ausentes.

2 Carpelos libres en la parte apical, por lo que los estilos se observan manifiestamente separados entre sí P. rugosa

2 Carpelos connados en un solo cuerpo; estilos conniventes.

3 Inflorescencia de aspecto tirsiforme, ramificada en la base P. thyrsiflora 3 Inflorescencia racemiforme o espiciforme, sin ramificaciones.

4 Inflorescencia racemiforme, laxa, flexible y a menudo ligeramente péndula, pedicelos de (4) 6 a $15 \mathrm{~mm}$ de largo; sépalos no acrescentes en fruto; planta característica de la franja del bosque mesófilo de montaña y de encinares muy húmedos de la región montañosa del este del país. P. americana

4 Inflorescencia espiciforme, densa, rígida y erecta, pedicelos de 0 a 4 (5) $\mathrm{mm}$ de largo; sépalos acrescentes en fruto; planta de amplia distribución geográfica y ecológica P. icosandra

\section{Phytolacca americana L.}

Esta es una planta renombrada en la literatura y conocida desde hace más de 200 años como componente común de la flora del este norteamericano. Siguiendo el criterio de Moquin-Tandon (1849), durante la mayor parte del siglo XIX se le llamó $P$. decandra L., pero a raíz del trabajo de Walter (1909) se está empleando el nombre más antiguo de $P$. americana. Varios autores han coincidido en opinar que en función de su androceo de 10 estambres, de su gineceo de 10 carpelos, de su inflorescencia en forma de racimo laxo, así como de su aislamiento geográfico, se trata de un taxon bien definido.

El primer indicio de su existencia en México lo constituyó la nota de Hemsley (1882), quien llamó la atención sobre el hecho de que un ejemplar colectado por E. Palmer en el estado de San Luis Potosí correspondía posiblemente a esta especie. 
En su obra monográfica, Walter (1909) describió como nueva a $P$. americana var. lancifolia con base en varios especímenes procedentes de la parte central de Veracruz y de otros dos de localidad incierta, posiblemente del estado de Hidalgo. Como características diferenciales de las plantas mexicanas, Walter mencionó sus flores más pequeñas, muy frecuentemente llevando 9 estambres y otros tantos carpelos, así como sus hojas lanceoladas o elípticas, acuminadas en ambos extremos.

Por su parte, Wilson (1932), en su contribución referente a la familia para North American Flora, redujo la mencionada variedad a sinonimia de $P$. americana, pero puso en duda la existencia de esta última en México.

No obstante, White (1940a, 1940b) y Marroquín (1968) citaron a $P$. americana de al menos cinco lugares con vegetación mesófila de la Sierra Madre Oriental de Nuevo León, mientras que Sharp et al. (1950) y Hernández X. et al. (1951) la encontraron como componente de un bosque húmedo de montaña en la misma Sierra Madre Oriental en el suroeste de Tamaulipas.

En su detallado trabajo sobre la distribución geográfica de $P$. americana, Sauer (1952) llegó a la conclusión de que el área natural de este taxon se extiende en forma continua desde el sureste de Canadá hasta la mitad oriental de Texas y también hasta Florida, con algunas localidades un tanto disyuntas, una de ellas cercana a la ciudad de Monterrey, en Nuevo León. En una llamada al pie, el mismo autor hizo referencia a las plantas de Veracruz consideradas por Walter como pertenecientes a la especie en cuestión, postulando la posibilidad de que en efecto constituyan una avanzada tropical aberrante y aislada de $P$. americana, pero indicando también la posibilidad de que se trate de individuos originados por hibridación entre otras especies de Phytolacca que prosperan en el mencionado estado.

Posteriormente Nowicke (1969), en su estudio palinotaxonómico de la familia, excluyó a México del área de la distribución conocida de $P$. americana, absteniéndose de comentar sobre el particular y al parecer sin encontrar otro acomodo a las plantas destacadas por Walter.

Es probable que las conclusiones de los dos últimos autores pesaron a su vez en la decisión de Martínez-García (1984) quien, en su fascículo correspondiente a la familia Phytolaccaceae para la Flora de Veracruz, tampoco aceptó la existencia de $P$. americana en el estado y a prácticamente todos los ejemplares de esta vinculación los determinó como $P$. purpurascens Kunth \& Bouché, especie cuyo tipo (probablemente destruido) proviene de Guatemala. De manera consecuente, con tal nombre aparecieron citadas plantas veracruzanas e hidalguenses en varios trabajos ulteriores.

De hecho, sin embargo, en el protólogo de $P$. purpurascens se describe un individuo con pedicelos más cortos que las flores, y éstas con 6 a 8 carpelos, características que discrepan en forma notable de los especímenes del este de México, pues en estos últimos las flores se observan conspicuamente pediceladas, y el número de carpelos varía entre (8)9 y 10(11).

Ante tal divergencia surgió la necesidad de volver a evaluar las plantas afines al grupo en cuestión y con tal propósito se examinaron 145 números de colecta (215 ejemplares), procedentes de 8 estados de la República y depositados en los principales herbarios nacionales. Tal conjunto de especímenes incluye casi todos los enumerados por MartínezGarcía (1984) bajo el nombre de $P$. purpurascens, además de otros, cuya relación aparece en el Apéndice de la presente contribución. 
El resultado de esta pesquisa fue en primera instancia algo desconcertante, pues se encontró que en las poblaciones mexicanas de Phytolacca caracterizadas por racimos laxos con pedicelos largos (de más de $8 \mathrm{~mm}$ en la fructificación), tépalos persistentes y carpelos en cantidad cercana a 10, el número de estambres no se mantiene estable. Así, si bien, en la mayor parte de los ejemplares de la parte central de Veracruz y de áreas adyacentes de Puebla se observaron 10 ó 9 en un solo verticilo, en algunas poblaciones de la misma región se contaron 12, 14 y hasta 16, dispuestos en dos series y con variaciones a veces en el mismo individuo. En uno de los conjuntos de localidades de Oaxaca, así como en la gran mayoría de los especímenes examinados de Hidalgo, los números hallados fueron de 18 y 20. En Querétaro y en puntos vecinos de San Luis Potosí, casi todos los individuos se caracterizaron por flores con 10 estambres, pero también se vieron algunas con 12. En las escasas localidades de Nuevo León y de Tamaulipas se contaron 10.

En cuanto al tamaño de las flores, también se registró una manifiesta variación, pues mientras en la región de Xalapa, Veracruz, prevalecen las de tamaño pequeño (tépalos de 1.5 a $2 \mathrm{~mm}$ de largo), en la mayor parte de las localidades son más bien de talla similar a la de las plantas de los Estados Unidos (tépalos de 2 a $2.5 \mathrm{~mm}$ de largo) y no faltaron algunas poblaciones con flores más grandes (tépalos de ca. $3 \mathrm{~mm}$ de largo).

Sin embargo, lo interesante del caso es que el conjunto estudiado representa una entidad notablemente congruente desde el punto de vista ecológico y geográfico.

De acuerdo con la información de las etiquetas, sin menoscabo de su procedencia mayoritaria de ambientes perturbados y de la vegetación secundaria, casi sin excepción las plantas están ligadas con el bosque mesófilo de montaña (tipo de vegetación que contiene una proporción importante de elementos comunes con los bosques caducifolios del este norteamericano) y con algunos bosques húmedos equivalentes o contiguos. A la vez, su área de distribución se define esencialmente a lo largo de la vertiente de barlovento de la Sierra Madre Oriental y de su prolongación hacia el sur, desde Nuevo León y Tamaulipas hasta el norte de Oaxaca, incluyendo algunas serranías aisladas que se levantan en la planicie costera adyacente (véase mapa de la Fig. 1). El intervalo altitudinal registrado es de 500 a $2600 \mathrm{~m}$.

El carácter de los racimos tirsiformes en la base, que con cierta frecuencia se ha encontrado en las poblaciones de $P$. americana, se observó también en uno de los ejemplares colectados en el estado de Hidalgo ( $R$. Hernández M. 7298).

Lo anterior conduce a la conclusión de que Phytolacca americana extiende su área de distribución al sector del este de México, donde sólo prospera en una zona ecológicamente análoga a la de la región oriental de los Estados Unidos y donde manifiesta una variación morfológica más amplia que la que usualmente se presenta más al norte. En este contexto, el conjunto de plantas mexicanas definitivamente no se circunscribe al concepto de $P$. americana var. lancifolia $\mathrm{H}$. Walt. y no resultaría apropiado el uso de tal trinomio.

Por otro lado, es pertinente tomar en cuenta la circunstancia de que $P$. americana se conoce como elemento introducido a diversas regiones del mundo, principalmente a zonas productoras de la uva, donde los frutos de individuos cultivados se usan para coadyuvar a la coloración de algunos vinos. En muchos de tales sitios la especie se ha naturalizado y prospera como maleza. En esta calidad se registra del oeste de Estados Unidos, de Europa, de Asia, de África y de Sudamérica. 
Rzedowski y Calderón de Rzedowski: Notas Sobre el Género Phytolacca en México

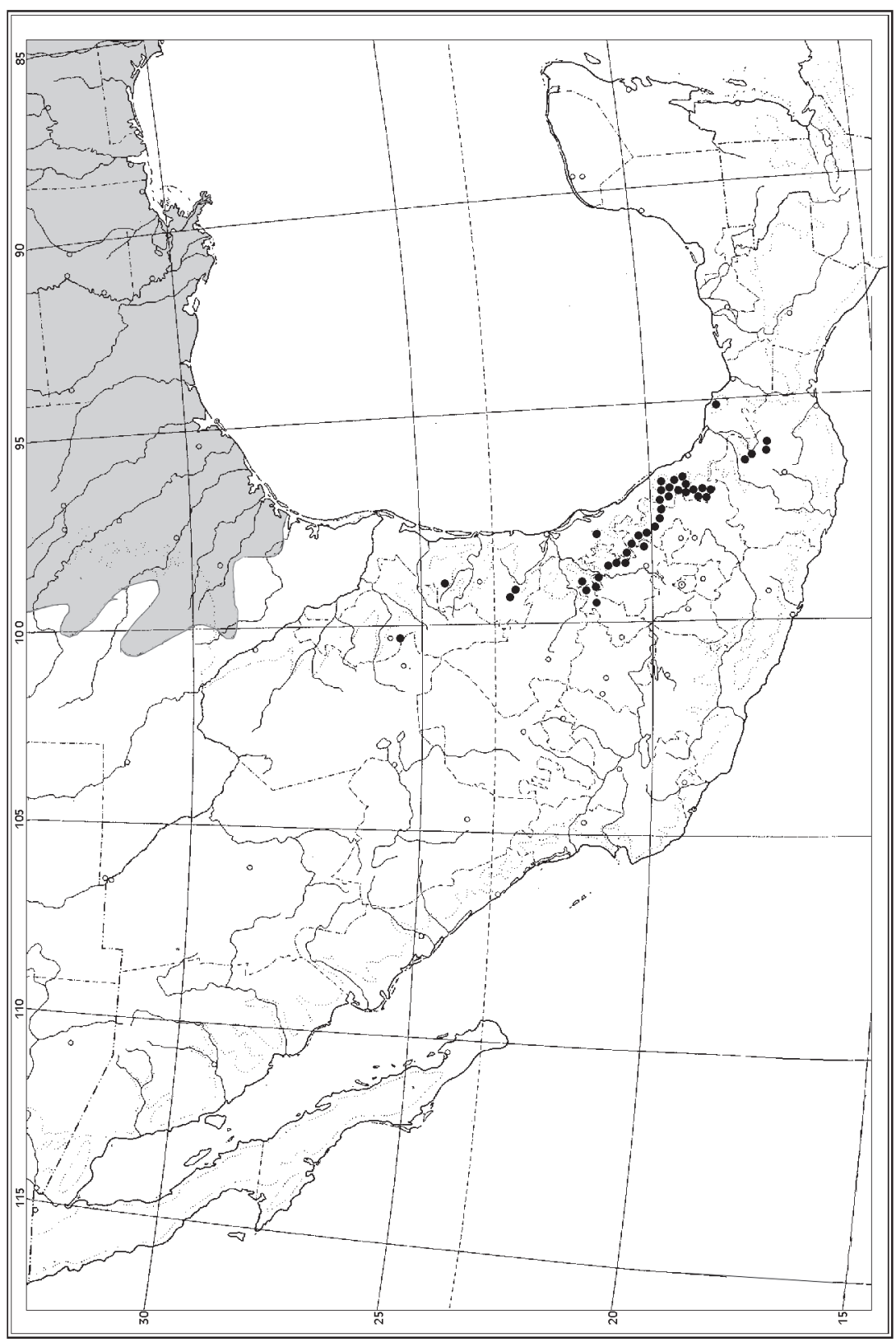

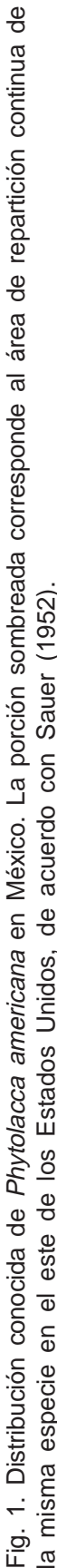


En teoría entonces, podría concebirse la idea de que la presencia de $P$. americana en México también se debe a una introducción. Con la información existente es imposible excluir por completo la realidad de tal evento, pero por su muy escasa probabilidad se pronuncian tanto la ausencia del cultivo de la planta en cuestión, al igual que del de la vid, en el oriente de este país, como también la variabilidad genética y la distribución geográfica de sus poblaciones mexicanas.

\section{Phytolacca heterotepala H. Walt.}

Bajo este nombre Walter (1909) describió plantas procedentes del centro de México, que discrepan de las demás especies de Phytolacca en su perianto algo zigomórfico, pues los tépalos difieren entre sí en forma y tamaño. En el protólogo se mencionan cinco ejemplares, varios de los cuales provienen del Distrito Federal y al menos dos proceden de colectas supuestamente mixtas.

Howell (1960) registró de terrenos baldíos y de jardines de la ciudad de San Francisco, la presencia de vegetales similares, aunque algunos caracterizados también por periantos de 6 a 8 tépalos e inflorescencias de tipo ramificado. Más recientemente plantas similares fueron encontradas por Ortiz (1989) en Portugal.

Nowicke (1969) pudo revisar el duplicado de uno de los sintipos de $P$. heterotepala y llegó a la conclusión que éste corresponde a $P$. octandra, pero al mismo tiempo confirmó la observación de Howell de que algunas colectas originarias de California coinciden con la descripción de Walter, por lo que aceptó la existencia de la especie en cuestión.

Sin embargo, en el conjunto de los materiales mexicanos de Phytolacca que se revisaron, se han visto unos pocos individuos (que en sus demás características coinciden con $P$. icosandra o a veces con $P$. rugosa), en los que todas o algunas flores presentan tépalos tangiblemente desiguales.

Este hecho, unido al reciente registro de diversas anomalías florales en una población de P. icosandra del Distrito Federal (Cruz Durán y Alcántara Ayala, 2000), hacen pensar que el taxon propuesto por Walter no corresponde sino a variantes ocasionales de la morfología floral.

\section{Phytolacca icosandra L. y P. octandra L.}

Estas dos entidades forman parte de un complejo, cuya interpretación ha sido controvertida. Mientras Moquin-Tandon (1849), Hemsley (1882), Walter (1909), Nowicke (1969), Wiggins (1980) y varios otros reconocen dos especies distintas, Wilson (1932), Standley (1937), Standley y Steyermark (1946), así como algunos autores mexicanos modernos las reúnen en una sola.

Ambas entidades presentan un área de distribución aproximadamente coincidente (desde México hasta la región andina de Ecuador y Perú), aunque la primera se registra también de las Antillas y como adventicia en California, mientras que la segunda se cita además de Asia, África, Australia y Oceanía. Es probable, sin embargo, que las localidades fuera del continente americano correspondan a plantas introducidas y naturalizadas. 
La separación clásica entre las dos se basa desde las descripciones originales en la diferencia en el número de estambres: 8 a 10 en un solo verticilo en $P$. octandra; 16 a 20 (a 22) en dos verticilos en $P$. icosandra). A ello se le agrega además el hecho de que la primera tiene inflorescencias más cortas que la segunda. No obstante, como ya lo observó Nowicke (1969), estos dos caracteres no siempre se encuentran correlacionados.

En México es notable que la gran mayoría de las plantas que prosperan en la mitad septentrional de la República responden en general bien a la definición de $P$. octandra. En el centro y en el sur del país, a su vez, se encuentran individuos que en forma más o menos sencilla pueden identificarse como pertenecientes a una o a otra especie, pero también con una participación muy cuantiosa de poblaciones que no quedan claramente ubicadas en ninguna.

Esta es la principal causa por la cual tanto Calderón de Rzedowski (1979), como Martínez-García (1984) y Lozada (2000) optaron por no diferenciar a P. octandra en sus respectivas áreas de estudio. El otro argumento de peso lo constituye el hecho de que no se ha podido observar una separación ecológica entre los dos supuestos taxa. Ambos tipos de plantas en México son habitantes comunes de áreas cuya vegetación original corresponde a bosques de coníferas, de encino y también a los mesófilos de montaña, usualmente entre 1200 y 3000 m de altitud. Poblaciones menos frecuentes, sin embargo, prosperan en regiones notablemente más secas (en medio de matorrales xerófilos) y también más cálidas (en los ambientes de bosques tropicales), descendiendo hasta lugares cercanos al nivel del mar.

Es razonable pensar que puede tratarse de dos especies originalmente bien delimitadas, pero que a causa de intensos disturbios ocasionados por las actividades del hombre han ido perdiendo su identidad en muchas comarcas.

Con más probabilidad se trata sólo de extremos de variación de un conjunto un tanto heterogéneo, en el cual se han ido seleccionando tendencias evolutivas divergentes.

Es factible asimismo que la realidad sea sensiblemente más compleja, pues así lo insinuaría la aparente gran tolerancia ecológica de $P$. icosandra en sentido amplio. El problema amerita una investigación profunda, pero al menos por el momento conviene adoptar el criterio conservador de la existencia de una sola especie variable, a la cual le corresponde el nombre más antiguo de $P$. icosandra.

\section{Phytolacca rivinoides Kunth \& Bouché}

Se trata de un taxon relativamente bien definido y delimitado. Destaca por sus inflorescencias racemosas, laxas y particularmente largas, frutos con mayor número (10 a 18) semillas, que consecuentemente son más pequeñas y más comprimidas que en la generalidad de las especies de Phytolacca, así como por la ausencia de tépalos en la fructificación avanzada.

Habita esencialmente en la vegetación secundaria derivada de bosques húmedos (tropical perennifolio, tropical subperennifolio y mesófilo de montaña) en un intervalo altitudinal de 0 a $1700 \mathrm{~m}$, con lo cual califica como la especie más termófila de las presentes en el país. En México se le ha registrado del centro de Veracruz y de áreas adyacentes de Hidalgo y Puebla a Chiapas, en la vertiente del Golfo de México y de Guerrero a Chiapas, 
del lado del Pacífico. Nowicke (1969) y Martínez-García (1984) señalan también su presencia en Nuevo León, pero no se han visto ejemplares que documenten esta avanzada.

Su distribución global se extiende en forma prácticamente continua hacia el sur a través de Centro y Sudamérica hasta Bolivia, Brasil, Paraguay y Argentina, incluyendo asimismo a las Antillas. En toda esta área muestra aparentemente afinidades ecológicas análogas.

\section{Phytolacca rugosa Braun \& Bouché}

Bajo este binomio, cuyo tipo procede de Guatemala, se ha estado reconociendo un conjunto de plantas procedentes de México, Centroamérica y Colombia, en las cuales el gineceo consiste de carpelos incompletamente fusionados en su parte apical.

Tal condición ha sido considerada como primitiva por la mayor parte de los autores y en consecuencia Walter (1909) ubicó a la especie en cuestión, junto con $P$. dioica L., $P$. chilensis Miers, $P$. sanguinea $\mathrm{H}$. Walt. y $P$. weberbaueri $\mathrm{H}$. Walt., que exhiben este mismo rasgo, en un subgénero separado que denominó Pircuniopsis.

La realidad muestra, sin embargo que, excepción hecha de este peculiar carácter, las plantas que se han estado identificando como $P$. rugosa difieren muy poco de las agrupadas en el subgénero Phytolacca, con las que frecuentemente conviven.

En Colombia, Fassett y Sauer (1950) revelaron la existencia de un enjambre híbrido entre $P$. rivinoides y $P$. rugosa en algunas áreas en que estas dos especies entran en contacto.

En Guatemala, Standley y Steyermark (1946) indicaron que $P$. rugosa está tan estrechamente relacionada con $P$. icosandra, que a veces es difícil de separar de esta última. En Veracruz, Martínez-García (1984) señaló que en algunas comarcas individuos de $P$. rugosa prosperan al lado de poblaciones de $P$. icosandra.

El examen de cerca de un centenar de especímenes procedentes de diferentes partes del territorio de México y que presentan el ovario, así como el fruto con los estilos evidentemente distanciados entre sí, revela la existencia de dos conjuntos algo diferentes y esencialmente alopátricos, aunque no siempre separados por su morfología en forma neta el uno del otro.

Los componentes del primero tienen inflorescencias claramente racemosas, de menos de $12 \mathrm{~cm}$ de largo y con pedicelos de (3)4 a 7(8) mm de largo. Sus flores llevan un número relativamente reducido de estambres (8 a 12) y sobre todo de carpelos ((4)5 a 8). Se distribuyen en forma discontinua y un tanto esporádica de Sinaloa y de Querétaro a Chiapas, entre 1000 y 2500 m de altitud, ligándose en forma al menos preferencial con el bosque mesófilo de montaña o con encinares y pinares francamente húmedos. Las características de estas plantas coinciden con las que formularon para $P$. rugosa Walter (1909), Nowicke (1969) en general, así como con las dadas para Costa Rica por Burger (1983) y para Guerrero por Lozada (2000).

El otro grupo incluye poblaciones con inflorescencias espiciformes hasta de $40 \mathrm{~cm}$ de largo, con pedicelos de 0 a 3(4) $\mathrm{mm}$ de longitud. Sus estilos suelen estar menos ampliamente separados entre sí, el número de estambres varía entre 8 y 22 y el de carpelos entre 7 y 10. Estas plantas tampoco presentan una distribución continua y su intervalo altitudinal es de 450 a $3300 \mathrm{~m}$. Frecuentemente conviven con P. icosandra (sensu lato), 
con la que comparten también la distribución geográfica, así como la tolerancia ecológica más amplias y no son pocos los individuos de características francamente intermedias y de dudosa asignación. Por otra parte, descuella la circunstancia de que en México estas plantas son los únicos representantes del género que se registran de altitudes superiores a $3000 \mathrm{~m}$. En la morfología de sus inflorescencias y flores el conjunto corresponde más de cerca a las descripciones dadas por Standley y Steyermark (1946) para Guatemala y por Martínez-García (1984) para Veracruz.

En primera instancia podría pensarse que las plantas del segundo grupo son el resultado de la influencia genética de $P$. rugosa (del primer conjunto) sobre $P$. icosandra y es factible que tal sea su génesis verdadera, pero el hecho de su simpatría comúnmente unilateral con esta última especie define una situación más complicada y difícil de interpretar.

Quedan pendientes de conocerse mejor la identidad y las relaciones filogenéticas de $P$. rugosa, así como su apropiada delimitación y ubicación taxonómica.

\section{Phytolacca thyrsiflora Fenzl ex J. A. Schmidt y P. sanguinea H. Walter}

En la literatura se ha estado reconociendo bajo el nombre de $P$. thyrsiflora a plantas con el pistilo de carpelos unidos, caracterizadas por una inflorescencia que al menos en su parte inferior se observa ramificada a manera de un tirso.

Poblaciones de este tipo prosperan a lo largo de amplias superficies de Brasil, extendiendo su área a la Guayana Francesa, a Perú y a Paraguay. Además, Walter (op. cit.) citó también una colecta de la República Dominicana, a la cual hacen referencia asimismo Wilson (op. cit.) y Liogier (1985), aunque aparentemente no se han vuelto a colectar plantas similares en la isla desde hace más de 140 años.

Martínez-García (1986) registró a su vez a $P$. thyrsiflora de México con base en cuatro ejemplares colectados en la península de Yucatán.

Al hacer la comparación de estos especímenes con varios procedentes del sur de Brasil, se encuentran suficientes semejanzas morfológicas para creer que pueden ser conespecíficos, por lo que procede, al menos tentativamente, considerar la presencia de la especie en nuestro país.

Surgen, sin embargo, profundas dudas al respecto, en virtud de las siguientes circunstancias:

a) los mencionados ejemplares de Campeche y Quintana Roo sólo parecen diferir en lo ramificado de sus inflorescencias de otros colectados en los mismos estados y determinados como $P$. icosandra;

b) entre los materiales del centro de México, donde es frecuente $P$. icosandra, se encontró también un espécimen de Aguascalientes (M. de la Cerda 5728 (IEB)), que presenta ramificaciones en la base de una de sus inflorescencias;

c) Howell (op. cit.) detectó este mismo carácter en plantas introducidas a California, que aquí se consideran como pertenecientes a $P$. icosandra (véase a este respecto el inciso correspondiente a $P$. heterotepala);

d) un ejemplar de la muestra B. Servín 542 (IEB), procedente de Querétaro que, en función de sus carpelos apicalmente separados, fue identificado como $P$. rugosa, presenta algunas ramificaciones en la base de una de sus inflorescencias y en tal virtud cabría 
adscribirlo a $P$. sanguinea, especie que reúne estas dos características, conocida de Colombia, pero también registrada de Venezuela y con duda (Burger, op. cit.; Nowicke, op. cit.) de Costa Rica; es interesante agregar que en otras réplicas de la mencionada colecta de Servín todas las inflorescencias se observan estrictamente racemosas;

e) Ya Nowicke (op. cit.) hizo ver que los racimos tirsiformes en la base se observan en forma esporádica en poblaciones de $P$. americana, así como en algunos ejemplares colectados en Asia, y entre los materiales revisados de México se registró además uno de $P$. rivinoides $(F$. Vázquez B. $482(\mathrm{XAL}))$ que presenta semejante arquitectura de la inflorescencia.

De lo anterior cabe deducir que la tendencia a ramificarse en la inflorescencia de Phytolacca constituye posiblemente un carácter recurrente de tipo atávico, cuyos elementos determinantes persisten en el genoma de muchas de las especies actuales del género, pero que sólo en algunas de las sudamericanas $(P$. sanguinea $\mathrm{H}$. y $P$. thyrsiflora) ejercen dominio del fenotipo, mientras que en otras el rasgo se manifiesta en forma ocasional o esporádica.

Esta compleja situación requiere indudablemente de un extenso y profundo análisis, con el objeto de lograr una disposición clasificatoria apropiada que defina, entre varios aspectos pendientes, la circunscripción de $P$. thyrsiflora frente a $P$. icosandra y a algunas otras especies, así como la de $P$. sanguinea frente a $P$. rugosa.

\section{Consideraciones finales}

Varios aspectos discutidos en los párrafos anteriores conducen a la formulación de algunas hipótesis, que cabría considerar en los trabajos taxonómicos futuros referentes a este grupo de plantas.

a) Desde los tiempos de Linneo (a quien se deben los binomios $P$. decandra, $P$. icosandra y $P$. octandra) y de otros botánicos del siglo XVIII y XIX, quienes agregaron $P$. dodecandra L'Hérit., $P$. heptandra Retz. y $P$. polyandra Batalin, la sistemática de Phytolacca descansó grandemente en la contabilidad del número de los estambres. En el trabajo fundamental de Walter (1909) no deja de prevalecer la misma tendencia, hecho que ha influido a su vez en los razonamientos e ideas de muchos otros autores.

Las observaciones que sirvieron de base al presente artículo llevan, sin embargo, a la postulación de la noción de que tales guarismos no siempre ni necesariamente funcionan como buen carácter para definir y separar especies de este género.

b) Una de las principales dificultades con la que se han tenido que enfrentar todos los intentos de un arreglo clasificatorio de las especies de Phytolacca es la escasez de "buenos" caracteres que permitan la separación y la correcta ubicación de los taxa en un sistema que refleje sus relaciones filogenéticas.

En esta luz, la idea propuesta en el párrafo anterior constituye una sustracción adicional al ya originalmente escaso arsenal de que disponen los estudiosos de la sistemática del género. Infortunadamente, en términos de morfología externa, los que escriben no tienen mucho de donde ofrecer para compensar tal merma.

Una ayuda importante, sin embargo, proviene de otra dirección, pues se revela que varias especies de Phytolacca, no obstante su manifiesta preferencia por los ambientes perturbados, muestran un buen grado de fidelidad respecto a determinados tipos de 
vegetación y pisos altitudinales. Tal vinculación se ha visto claramente en México; aunque hay poca información precisa sobre el particular, aparentemente también existe en América del Sur. De esta suerte, el aspecto de las afinidades ecológicas, en combinación con la repartición geográfica de los taxa, amerita tomarse en cuenta en el proceso de su apropiada definición.

Una novedad adicional que no deberá soslayarse tampoco en los trabajos futuros es el conocimiento de la incidencia de anomalías florales en el género. Por una parte convendrá saber más acerca de los factores que favorecen su génesis, así como de sus alcances y modalidades, en particular si la arquitectura de la inflorescencia también está sujeta a variantes de este tipo. Será recomendable asimismo revisar hasta qué punto tal fenómeno pudo haber afectado la taxonomía existente para el grupo y eventualmente realizar los ajustes necesarios.

c) La distribución actual de Phytolacca mucho tiene que ver con dos factores extrínsecos: el hombre y las aves. Abstracción hecha en esta discusión de la influencia humana, no hay mucha duda de que los pájaros son los principales responsables de la dispersión de frutos y semillas de estas plantas, pues al respecto existen claros indicios, así como varias referencias en la literatura.

A su vez, la presencia de Phytolacca en las islas oceánicas, como las Hawai y las Galápagos, permite suponer que las aves migratorias deben haber contribuido en forma significativa a la expansión de las áreas de repartición geográfica de muchas de sus especies y del conjunto en su totalidad, actuando como vectores de transporte de propágulos a mediana y larga distancia.

La realidad de tal mecanismo daría la explicación del hecho de que ciertas especies morfológicamente muy variables (como $P$. americana y $P$. icosandra), en algunas de sus áreas parciales un tanto disyuntas o marginales sólo muestren una fracción de su variabilidad genética conocida.

d) En el caso concreto de $P$. americana cabe pensar en su posible origen mexicano, pues es allí donde se concentra su actual variabilidad. Es factible que esta especie migró al norte y ocupó extensas porciones del este de Estados Unidos quizás en tiempos relativamente recientes ()finales del Terciario o principios del Cuaternario?), cuando los vaivenes climáticos pueden haber propiciado el desplazamiento.

Es de considerarse que tal invasión sólo pudo haber tenido éxito si iba acompañada de la simultánea adquisición de la resistencia a prolongadas exposiciones a temperaturas inferiores a $0^{\circ} \mathrm{C}$ por parte de las raíces de las plantas. A este respecto no sería ilógico especular que algunas poblaciones mexicanas podían haber estado preadaptadas a un requerimiento semejante.

La probabilidad de este movimiento migratorio se ajustaría al modelo postulado por McVaugh (1952) para otros linajes de plantas vasculares. Sería también consistente con la idea de que la evolución y diversificación de Phytolacca y plantas afines puede haber tenido lugar primariamente en las porciones montañosas de Sudamérica y que su desplazamiento hacia otras regiones debe haber sido cronológicamente posterior.

En cuanto al origen mismo de $P$. americana, cabe tomar en cuenta la sugestión de Sauer (1952) de que se trata de una entidad generada por hibridación. De ser así, como los progenitores más viables pueden haber operado $P$. rivinoides y $P$. icosandra, especies de amplia presencia en el este de México. 


\section{AGRADECIMIENTOS}

Se agradece al M. en C. Emmanuel Pérez Calix su significativa ayuda en la confección del mapa, así como a la Biól. Julieta Martínez-García y al M. en C. Sergio Avendaño la información proporcionada sobre algunos aspectos bibliográficos.

\section{LITERATURA CITADA}

Burger, W. 1983. Phytolaccaceae. In: Flora Costaricensis. Fieldiana, Bot. n.s. 13: 199-212.

Calderón de Rzedowski, G. 1979. Phytolaccaceae. Flora fanerogámica del Valle de México 1: 160-161.

Cruz Durán, R. y O. Alcántara Ayala. 2000. Anormalidad floral en Phytolacca icosandra L.

(Phytolaccaceae) en el Pedregal de San Angel, México, D. F. Acta Bot. Mex. 53: 27-33. 2000.

Fassett, N. y J. D. Sauer. 1950. Studies of variation in the weed genus Phytolacca. I. Hybridizing species in northeastern Colombia. Evolution 4: 332-339.

Hemsley, W. B. 1882. Phytolacca. In: Botany. Biologia Centrali-Americana. R. H. Porter. Londres. vol. 3. pp. 29-30.

Hernández X., E., H. Crum, W. B. Fox y A. J. Sharp. 1951. A unique vegetational area in Tamaulipas. Bull. Torr. Bot. Club 78: 458-463.

Howell, J. T. 1960. A Mexican pokeberry in San Francisco, California. Leafl. West. Bot. 9: 81-83.

Liogier, A. H. 1983. Phytolaccaceae. In: La flora de la Española. II. Universidad Central del Este. Serie Científica XV. San Pedro de Macorís, Rep. Dominicana. pp. 130-142.

Lozada, L. 2000. Phytolaccaceae. Flora de Guerrero 10: 1-20.

Marroquín, J. S. 1968. Datos botánicos de los cañones orientales de la Sierra de Anáhuac, al sur de Monterrey, N.L., México. Cuads. Inst. Invs. Ciens. Univ. N.L. Méx. 14: 1-79.

Martínez-García, J. 1984. Phytolaccaceae. Flora de Veracruz 36: 1-41.

Martínez-García, J. 1986. Notas del herbario XAL. V. Phytolacca thyrsiflora Fenzl (Phytolaccaceae), nuevo registro para México. Biotica 11: 181-182.

McVaugh, R. 1952. Suggested phylogeny of Prunus serotina and other wide ranging phylads in North America. Brittonia 7: 317-346.

Moquin-Tandon, A. 1849. Phytolaccaceae. In: A. DC. Prodr. 13(2): 1-40, 459-460.

Nowicke, J. W. 1969. Palynotaxonomic study of the Phytolaccaceae. Ann. Mo. Bot. Gard. 55: 294-363.

Ortiz, S. 1989. Una nueva especie de fitolaca adventicia en Europa: Phytolacca heterotepala H. Walter. Anuario Soc. Broter. 54: 15-18.

Sauer, J. D. 1952. A geography of pokeweed. Ann. Mo. Bot. Gard. 39: 113-125.

Sharp, A. J., E. Hernández Xolocotzi, H. Crum y W. B. Fox. 1950. Nota florística de una asociación importante del suroeste de Tamaulipas, México. Bol. Soc. Bot. Méx. 11: 1-4.

Standley, P. C. 1937. Phytolaccaceae. In: Flora of Costa Rica. Field Mus. Nat. Hist. Bot. Ser. 18: 426428.

Standley, P. C. y J. A. Steyermark. 1946. Phytolaccaceae. In: Flora of Guatemala. Fieldiana, Bot. 24(4): 192-202.

Walter, H. 1909. Phytolaccaceae. Pflanzenr. IV. 83 (39): 1-154.

White, S. S. 1940a. Flora of hacienda Vista Hermosa, Nuevo León. Papers Mich. Acad. 26: 81-86.

White, S. S. 1940b. Vegetation of Cerro de la Silla, near Monterrey, Mexico. Papers Mich. Acad. 26: 87-98.

Wiggins, I. L. 1980. Phytolaccaceae. In: Flora of Baja California. Stanford University Press. Stanford, Calif. pp. 150-154.

Wilson, P. 1932. Petiveriaceae. North Amer. Flora 21: 257-266. 
Rzedowski y Calderón de Rzedowski: Notas Sobre el Género Phytolacca en México

\section{APÉNDICE}

Ejemplares examinados de Phytolacca americana de México (adicionales a los enumerados en el fascículo 36 de la Flora de Veracruz como pertenecientes a $P$. purpurascens).

Nuevo León: municipio de Santiago, arroyo "Cola de Caballo", a la altura de la caída del agua, J. M. López y N. Moreno P. 287 (ENCB, MEXU); municipio de Santiago, Cola de Caballo, G. B. Hinton et al. 24115 (IEB); municipio de Santiago, La Nogalera, G. B. Hinton et al. 24408 (IEB, MEXU).

Tamaulipas: municipio de San Carlos, $8 \mathrm{~km}$ al $\mathrm{W}$ de San Carlos, en el camino al Diente, M. Martínez y J. Martínez 2319 (XAL); municipio de San Carlos, Cerro del Diente, Sierra de San Carlos, $17 \mathrm{~km}$ al SW de San Carlos, L. Hernández 1322 (MEXU); municipio de San Carlos, Sierra de San Carlos, en el Cerro del Diente, M. Martínez 774 (MEXU); municipio de San Carlos, Cerro del Diente en la Sierra de San Carlos, M. Martínez 1095 (ENCB; MEXU); municipio de Gómez Farías, $1 \mathrm{~km}$ al SE del rancho Julilo y $16 \mathrm{~km}$ al NE de Gómez Farías, F. González Medrano et al. 3628 (MEXU); municipio de Gómez Farías, entre Alta Cima y Rancho del Cielo (Sierra de Guatemala), F. González Medrano et al. 4261 (MEXU); municipio de Gómez Farías, 4 km al NW de Gómez Farías, M. Yánez 423 (ENCB, MEXU).

San Luis Potosí: municipio de Aquismón, Tampaxal, J. B. Alcorn 3054 (MEXU).

Querétaro: municipio de Jalpan, 4-5 km al oriente de La Parada, B. Servín 259 (IEB); municipio de Landa, Puerto de los Cajones, aprox. $4 \mathrm{~km}$ al noroeste de La Yesca, E. González Ponce 313 (IEB); municipio de Landa, $1 \mathrm{~km}$ al noroeste de El Humo, H. Rubio 312 (IEB); municipio de Landa, $5 \mathrm{~km}$ al S de El Lobo, sobre el camino a Agua Zarca, J. Rzedowski 42458 (ENCB, IEB, XAL); municipio de Landa, $10 \mathrm{~km}$ al SE de Agua Zarca, sobre el camino a Pisaflores, J. Rzedowski 43306 (ENCB, IEB, MEXU, XAL); municipio de Pinal de Amoles, aprox. 5 km de El Llano, camino a San Pedro Escanela, E. Carranza 880 (IEB); municipio de Pinal de Amoles, río Escanelilla, $1 \mathrm{~km}$ al $\mathrm{S}$ de Escanelilla, sobre la carretera a Pinal de Amoles, E. Pérez et al. 3736 (IEB).

Hidalgo: municipio de Chapulhuacán, ca. $18.1 \mathrm{mi}$ NE of Jacala on route 85 towards Tamazunchale, $99^{\circ} 10^{\prime} \mathrm{W}, 2^{\circ} 05^{\prime} \mathrm{N}$, alt. $1650 \mathrm{~m}$, J. A. Soule 2915 (ENCB, MEXU); municipio de Tlanchinol, $3.1 \mathrm{~km}$ SW of Tlanchinol on highway 105, B. Bartholomew et al. 3438 (MEXU); municipio de Lolotla, $7 \mathrm{~km}$ al NE de Lolotla, sobre la carretera Pachuca - Tampico, S. Zepeda A. 27 (IEB, XAL); municipio de Eloxochitlán, predio El Alcalaque, $2 \mathrm{~km}$ al NW del poblado Eloxochitlán, J. L. López García 432 (IEB, MEXU); municipio de Molango, paraje Ayoco, J. L. López-García 21 (IEB, MEXU); municipio de Xochicoatlán, $3 \mathrm{~km}$ al S de Jalamelco, S. Acosta y M. A. Barrios 318 (ENCB, IEB, MEXU); municipio de Tianguistengo, $8 \mathrm{~km}$ al E de Tianguistengo, hacia Pemuxco, R. Hernández M. 3976 (MEXU); municipio de Zacualtipán, paraje Cumbre de Tlahuelompa, $2 \mathrm{~km}$ al SW del ejido de Tlahuelompa, J. L. López García 379 (IEB, MEXU); municipio de Zacualtipán, Tlahuelompan, $12 \mathrm{~km}$ al sur de Zacualtipán, R. Hernández M. y P. Tenorio L. 7084 (ENCB, MEXU); municipio de Metztitlán, 
Zoquizoquiapan, $5 \mathrm{~km}$ al $\mathrm{N}$ de Alumbres, R. Hernández M. et al. 5829 (ENCB); municipio de Tenango de Doria, camino Tenango de Doria - La Viejita, E. Turra 1189 (ENCB); municipio de Tenango de Doria, $3 \mathrm{~km}$ al E de Tenango de Doria, hacia El Cirio, R. Hernández M. 7298 (IEB, MEXU); municipio de Tenango de Doria, $5 \mathrm{~km}$ al este de Tenango de Doria, hacia El Cirio, R. Hernández M. et al. 4155 (ENCB, MEXU); municipio de Tenango de Doria, entre Metepec y Tenango de Doria, $30 \mathrm{~km}$ al NW de Tulancingo y $21 \mathrm{~km}$ al NNW de Metepec, J. Espinoza H. 54 (ENCB); municipio de Acaxochitlán, San Francisco, A. Villa Kamel 105 (IEB).

Puebla: municipio de Huauchinango, Huauchinango, cerca de la carretera México Huauchinango, 29.VIII.1974, A. Patiño 27 (ENCB); municipio de Huauchinango, near Catalina, Huauchinango, A. J. Sharp 441155 (MEXU); municipio de Amixtlán, Amixtlán, camino, 2040' N, 9747' W, S. Vargas 296 (XAL); municipio Xochitlán, Xochitlán de Vicente Suárez, 1958' N, 97044' W, G. Villalobos 331 (MEXU); municipio de Zacapoaxtla, cañada del río Apulco, al N de Zacapoaxtla, M. A. Martínez A. et al. 87 (MEXU); municipio de Teziutlán, Teziutlán, E. Hernández X. X-67 (MEXU).

Veracruz: municipio de Tepetzintla, Sierra de Otontepec, al NE de Tepetzintla, $21^{\circ} 16$ N, 97052' W, F. Vázquez y F. Tapia 197 (XAL); municipio de Huayacocotla, along Huayacocotla - Zontecomatlán road, between Miguel Lerdo and Antonio Ixtatela, $3 \mathrm{~km} \mathrm{NE}$ of Miguel Lerdo, 2040'30" N, 98²4 W, M. Nee y K. Taylor 26848 (XAL); municipio de Huayacocotla, along Huayacocotla - Zontecomatlán road, between Barro Colorado and Los Tepozanes, $2 \mathrm{~km}$ by road NE of Agua de La Calabaza and $5 \mathrm{~km}$ by road SW of Zitacatipan, 20³8' N, 98²7' W, M. Nee y K. Taylor 26878 (XAL); municipio de Tlapacoyan, about 6 km (by air) S of Tlapacoyan, on road to Altotonga, 1955' N, 97013' W, M. Nee y G. Diggs 24872 (XAL); municipio de Atzalan, between "Puente de Rieles" and "La Calavera", $9.5 \mathrm{~km}$ (by raod), ca. $6 \mathrm{~km}$ by air) NE of Altotonga on road to Tlapacoyan, 1949' N, 97013' W, M. Nee y G. Diggs 24895 (XAL); municipio de Chiconquiaco, $500 \mathrm{~m}$ al N del poblado de Chiconquiaco, $19^{\circ} 45$ N, 97²9' W, C. Gutiérrez B. 3392 (XAL); municipio de Chiconquiaco, 6 km aprox. al NE de Chiconquiaco, camino a Vaquería, 1944' N, 9648' W, I. Pérez y L. Tapia 404 (XAL); municipio de Chiconquiaco, en el poblado de Planta El Pie a Loma Alta, entrada por Chiconquiaco, 19॰44' N, 9648' W, J. I. Calzada et al. 9382 (ENCB, MEXU, XAL); municipio de Villa Aldama, $10 \mathrm{~km}$ antes de llegar al poblado de Villa Aldama, 19³9' N, 97'13' W, J. Martínez-García 36 (MEXU, XAL); municipio de Las Minas, Rinconada, por el cerro de la Tolda, al pie de la barranca, 19³9' N, 9708' W, C. Durán y E. Garibay 474 (XAL); municipio de Naolinco, $6 \mathrm{~km}$ ENE of Naolinco on the road to Misantla (Hwy 136), E. Judziewicz et al. 3119 (MEXU, XAL); municipio de Naolinco, El Órgano, 5 km antes de Naolinco, carretera Xalapa - Misantla, municipio de Naolinco, 1940' N, 9654' W, L. Tapia y F. Vázquez 390 (XAL); en el mirador de la cascada de Naolinco, 1940' N, 9652' W, J. I. Calzada 9409 (ENCB, MEXU, XAL); municipio de Tlacolulan, carretera de San Miguel a Tlacolulan, 19³7' N, 9658' W, F. Vázquez 2117 (XAL), 2127 (XAL); municipio de Acajete, km 2 camino La Joya - Joya Chica, 19³6' N, 9701' W, H. Narave F. y N. Moreno 862 (MEXU, XAL); municipio de Acajete, entrada a Plan de Sedeño, 19॰34' N, 970' W, P. Zamora y J. López P. 3863 (XAL); municipio de Rafael Lucio, carretera Banderilla - Perote, rancho Santa Bárbara, 19³7' N, 9659' W, F. Vázquez 1869 (XAL); municipio de Banderilla, tramo Banderilla - Jilotepec, vía de ferrocarril, P. Padilla S. 38 (ENCB); municipio de Banderilla, 
rancho La Mesa, a 1 km aprox. de Banderilla, 19³5'00" N, 9658'00" W, I. Pérez y L. Mendizábal 304 (XAL); municipio de Banderilla, a 2 km de Banderilla, carretera a México, poblado El Rosario, rancho El Balconcito, 19³6' N, 9557' W, M. Lascurain 89 (XAL); municipio de San Andrés Tlalnehuayocan, $2 \mathrm{~km}$ de Tlalnehuayocan, con dirección a Mazatepec, del lado norte del camino de terracería, 19³4'30" N, 9658'40" W, P. Zamora C. 2852 (XAL); municipio de San Andrés Tlalnehuayocan, $1 \mathrm{~km}$ above and NW of San Andrés Tlalnehuayocan, M. Nee et al. 26199 (XAL); municipio de San Andrés Tlalnehuacoyan, Otilpan, 19³3' N, 9658' W, C. Gutiérrez 4144 (XAL); municipio de Xalapa, El Seminario, al O de la ciudad de Xalapa, 19³2' N, 9657' W, C. Gutiérrez B. 1520 (ENCB, IEB, XAL); municipio de Xalapa, ciudad de Xalapa, B. A. Domínguez 15 (XAL); municipio de Xalapa, cerro de Macuiltepec, M. G. Zolá 715 (XAL); municipio de Xalapa, Parque Ecológico Francisco Javier Clavijero, 19³1' N, 965' W, J. Zavaleta 46 (XAL); municipio de Coatepec, Loma Alta, 19³0'15" N, 97²'30" W, V. E. Luna M. 998 (XAL); municipio de Coatepec, entre Coatepec y Zaragoza, rumbo a los tanques de agua, 19²8' N, 96 ${ }^{\circ} 9^{\prime}$ W, P. Zamora 2492 (XAL); municipio de Coatepec, Coatepec Viejo, 19²7'7" N, 970'15" W, V. E. Luna 1362 (XAL); municipio de Xico, fondo de la barranca El Caracol, 19³1' N, 97 $06^{\prime} \mathrm{W}, \mathrm{H}$. Narave y F. Vázquez 426 (XAL); municipio de Xico, Matlalapa, 19²9' N, 9706' W, H. Narave et al. 219 (IEB, XAL); municipio de Ixhuacán de Los Reyes, $2.5 \mathrm{~km}$ (by road) E of Ayahualulco and $1.6 \mathrm{~km}$ (by road) W of Ixhuacán de Los Reyes, 19²2' N, 9708' W, M. Nee 22958 (XAL); municipio de Ixhuacán de Los Reyes, adelante de El Arenal, camino a Ixhuacán, $F$. Vázquez 1826 (XAL); municipio de Teocelo, Teocelo, 19²7'7"N, 9658'8" W, R. Cervantes M. 16 (XAL), 32 (XAL); 47 (XAL); 59 (XAL); municipio de Teocelo, Teocelo, B. Guerrero 1760 (XAL); municipio de Calcahualco, entrada por Coscomatepec, faldas del Pico de Orizaba, 19¹0' N, 9703' W, F. Vázquez 1710 (XAL); municipio de Calcahualco, Acomulco, vereda hacia puente Don Porfirio, barranca del río Jamapa, 1909' N, 9707' W, J. L. Martínez y F. Vázquez 684 (IEB, MEXU, XAL); municipio de Calcahualco, Rincón Atotonilco, camino al Cajón, 1908' N, 97013' W, J. L. Martínez y F. Vázquez 1159 (IEB, XAL); municipio de Calcahualco, $1 \mathrm{~km}$ al $\mathrm{O}$ de Escola, camino al Banco, 1908' N, 9706' W, J. L. Martínez 33 (MEXU, XAL); municipio de Calcahualco, $500 \mathrm{~m}$ antes de llegar a Calcahualco, 1908' N, 9705' W, T. Mejía-Saulés y F. Vázquez B. 1172 (IEB, MEXU, XAL); municipio de Calcahualco, a $2 \mathrm{~km}$ de Ahuihuixtla, camino a Tres Aguas (Coscomatepec), 1908' N, 9703' W, J. L. Martínez y F. Vázquez 273 (XAL); municipio de Calcahualco, Ahuihuixtla, $3 \mathrm{~km}$ camino a Calcahualco, barranca del río Jamapa, $19^{\circ} 08^{\prime} \mathrm{N}, 9^{\circ} 03^{\prime} \mathrm{W}, \mathrm{J}$. Martìnez y F. Vázquez 800 (IEB, MEXU, XAL); $5 \mathrm{~km}$ antes de Calcahualco, municipio de Coscomatepec, 19¹0' N, 9659' W, F. Vázquez 359 (XAL); municipio de Chocamán, cerro de Chocamán, cerca del poblado de Chocamán, 1901' N, 9703' W, T. Mejía et al. 1248 (XAL); municipio de Mariano Escobedo, Ocosotla hacia Tesmalaca, camino de terracería, R. I. Aguilar et al. 105 (IEB, MEXU); municipio de Maltrata, alrededores de Maltrata, 1849' N, 97017' W, F. Vázquez B. 871 (ENCB, IEB, MEXU, XAL); municipio de Tequila, 4 km de la carretera Tequila a Orizaba, 1843' N, 9705' W, F. Vázquez B. 1404 (ENCB, IEB, MEXU, XAL); municipio de Zongolica, 2 km al SE de Zongolica, 1844' N, 9655 W, F. Vázquez 1830 (XAL); municipio de Zongolica, Tlanecpakila, M. E. Ventura 219 (XAL); municipio de Zongolica, entre Zongolica y Los Reyes, F. Vázquez 1369 (XAL); municipio de Texhuacán, San Juan - Texhuacán, 18³7'6" N, 97²'18" W, C. Weimann 160 (XAL). 
Oaxaca: municipio de Comaltepec, km 105, entre Llano de las Flores y Tuxtepec, C. Delgadillo M. 98 (MEXU); municipio de Comaltepec, approx. $13 \mathrm{~km} \mathrm{~N}$ of desviación to Yolox, G. J. Martin 478 (ENCB, MEXU); municipio de Comaltepec, atlantic slope of mountains, along rd. between Ixtlan and Valle Nacional, G. Breckon y B. Christman 673 (MEXU); municipio de Comaltepec, brecha 60, ca. $7 \mathrm{~km}$ al $\mathrm{N}$ de la desviación a Yolox, $D$. H. Lorence et al. 3226 (ENCB, MEXU, XAL); municipio de Comaltepec, $3 \mathrm{~km}$ al S de Metates, carretera Tuxtepec - Oaxaca, R. Torres C. y L. Cortez A. 7275 (MEXU); municipio de Comaltepec, $2 \mathrm{~km}$ al $\mathrm{S}$ de la carretera Vista Hermosa - Esperanza, C. Sánchez A. 128 (ENCB, MEXU); municipio de Macuiltianguis, brecha 200; terrenos forestales de la comunidad de San Pablo Macuiltianguis, E. Guízar 751 (IEB); municipio de Macuiltianguis, en la brecha 200, a la altura del "Caracol", G. Pérez Cruz (C17) (ENCB); municipio de Macuiltianguis, sobre la brecha 200 a la entrada de la 280, E. Pérez Portilla B26 (ENCB, $\mathrm{XAL}$ ); municipio de Macuiltianguis, San Pablo Macuiltianguis, a $10 \mathrm{~km}$ de la entrada por la Puerta del Sol, 17033' N, 96³3' W, J. I. Calzada 5038 (IEB, XAL); municipio de Melchor Betaza, $2 \mathrm{~km}$ al SE de la desviación a Oaxaca, por Mixes; la desviación está a $3.5 \mathrm{~km}$ al S de San Andrés Yaá, R. Torres y R. Cedillo 2034 (ENCB); municipio de San Andrés Yaá, $26 \mathrm{~km}$ al $\mathrm{N}$ de Yacochi, en el camino a San Andrés Yaá, distrito Mixe, R. Torres C. et al. 7078 (MEXU); municipio de Totontepec, Santiago Amatepec, $17^{\circ} 15$ N, 9600' W, W. Ruiz 64 (MEXU); municipio de Totontepec, $2 \mathrm{~km}$ sobre la terracería Totontepec - Villa Alta, distrito Mixe, A. García-Mendoza y R. Torres 2019 (MEXU); municipio de Totontepec, along road between Mitla and Zacatepec, $40 \mathrm{~km}$ by road ENE of Ayutla, $10 \mathrm{~km}$ by road $\mathrm{E}$ of intersection with road to Totontepec, $17^{\circ} 08^{\prime}$ N, 96 $03^{\circ}$ W, G. Diggs et al. 3910 (XAL). 